\title{
Preventive Effects of the Dietary Intake of Medium-chain Triacylglycerols on Immobilization-induced Muscle Atrophy in Rats
}

\author{
Shuhei Nishimura ${ }^{1}$, Makoto Inai ${ }^{1}$, Tetsuo Takagi ${ }^{2}$, Yudai Nonaka', Shogo Urashima ${ }^{1}$, \\ Kazumitsu Honda ${ }^{2}$, Toshiaki Aoyama ${ }^{2}$ and Shin Terada ${ }^{1, *}$ \\ ${ }^{1}$ Department of Life Sciences, Graduate School of Arts and Sciences, The University of Tokyo, 3-8-1 Komaba, Meguro-ku, Tokyo 153-8902, \\ JAPAN \\ ${ }^{2}$ Central Research Laboratory, The Nisshin-OilliO Group, Ltd., 1 Shinmori-cho, Isogo-ku, Yokohama, Kanagawa 235-8558, JAPAN
}

\begin{abstract}
Previous studies have shown that medium-chain triacylglycerols (MCTs) exert favorable effects on protein metabolism. This study evaluated the effects of the dietary intake of MCTs on rat skeletal muscle mass and total protein content during casting-induced hindlimb immobilization, which causes substantial protein degradation and muscle atrophy. Rats were fed a standard diet containing long-chain triacylglycerols (LCTs) or MCTs for 3 days and then a unilateral hindlimb was immobilized while they received the same diet. After immobilization for 3, 7, and 14 days, muscle mass and total protein content in immobilized soleus muscle in the LCT-fed rats had markedly decreased compared to the contralateral muscle; however, these losses were partially suppressed in MCT-fed rats. Autophagosomal membrane proteins (LC-I and -II), which are biomarkers of autophagy-lysosome activity, did not differ significantly between the LCT- and MCT-fed rats. In contrast, the immobilization-induced increase in muscle-specific E3 ubiquitin ligase MuRF-1 protein expression in immobilized soleus muscle relative to contralateral muscle was completely blocked in the MCT-fed rats and was significantly lower than that observed in the LCT-fed rats. Collectively, these results indicate that the dietary intake of MCTs at least partly alleviates immobilization-induced muscle atrophy by inhibiting the ubiquitin-proteasome pathway.
\end{abstract}

Key words: medium-chain triacylglycerols, skeletal muscle, immobilization, ubiquitin-proteasome pathway

\section{INTRODUCTION}

Skeletal muscle constitutes around $40 \%$ of the human body by mass and participates in locomotion, production of body heat, metabolic regulation, and other functions. Prolonged periods of skeletal muscle inactivity caused by immobilization, denervation, bed rest, or microgravity lead to considerable loss of muscle mass and myofibrillar protein content. Muscle atrophy results in impaired exercise performance, poor health, and reduced quality of life. Therefore, the development of an effective treatment to mitigate inactivity-induced muscle atrophy is required. As muscle atrophy is caused by an imbalance between the rates of protein synthesis and degradation, treatment that could improve protein metabolism may help to prevent the progression of inactivity-induced muscle atrophy.

Medium-chain fatty acids (MCFAs), which consist of chains of 8-10 carbon atoms, have several unique proper- ties in comparison with long-chain fatty acids $(\mathrm{LCFAs})^{1,2)}$. Unlike the majority of other dietary fats that are rich in LCFAs, medium-chain triacylglycerols (MCTs), which are composed exclusively of MCFAs, are hydrolyzed rapidly. The resultant MCFAs are absorbed directly by the liver via the portal vein and oxidized easily. It has been well documented that MCTs have anti-obesity and anti-diabetic effects $^{2-4)}$. In addition, several lines of evidence suggest that MCTs exert favorable effects on protein metabolism; for example, alterations in body weight and nitrogen balance were found to be more favorable in surgical patients fed MCTs parenterally than in those who received long-chain triacylglycerols (LCTs) ${ }^{5}$. Recent studies have also demonstrated that the dietary intake of MCTs improved serum albumin concentrations in aged individuals with protein-energy malnutrition and in rats fed a low-protein $\operatorname{diet}^{6-8)}$. These results led us to hypothesize that the

*Correspondence to: Shin Terada, Department of Life Sciences, Graduate School of Arts and Sciences, The University of Tokyo, 3-8-1 Komaba, Meguro-ku, Tokyo 153-8902, JAPAN

E-mail: terada@idaten.c.u-tokyo.ac.jp

Accepted April 10, 2017 (received for review March 6, 2017)

Journal of Oleo Science ISSN 1345-8957 print / ISSN 1347-3352 online

http://www.jstage.jst.go.jp/browse/jos/ http://mc.manusriptcentral.com/jjocs 
consumption of MCTs could also be a useful treatment to alleviate inactivity-induced muscle atrophy. In this context, the present study was undertaken to evaluate the effects of the dietary intake of MCTs on muscle mass and muscle protein content in rats subjected to casting-induced hindlimb immobilization, which is the most commonly used model of inactivity-induced muscle atrophy ${ }^{9,10)}$.

As mentioned above, skeletal muscle atrophy can be induced by an imbalance between the rates of protein synthesis and degradation. Increased protein degradation is a major factor in muscle atrophy induced by reduced mechanical loading, whereas there is not always a reduction in protein synthesis ${ }^{11)}$. Skeletal muscle utilizes two major protein degradation pathways: the ubiquitin-proteasome pathway, which plays an important role in selective degradation of proteins ${ }^{12)}$, and the autophagy-lysosome pathway, which forms an intracellular bulk degradation system ${ }^{13}$. The second aim of this study, if the dietary intake of MCTs was found to mitigate immobilized-induced muscle atrophy, was to evaluate the effects of MCTs on these degradation pathways in skeletal muscle and to elucidate the mechanisms mediating the prevention of muscle atrophy by MCTs.

\section{EXPERIMENTAL}

\subsection{Animal treatments}

Animal treatment was conducted as reported previous$\mathrm{ly}^{14)}$. Eight-week-old male Wistar rats (CLEA Japan, Inc., Tokyo, Japan) were housed individually in cages. The animal room was maintained at $23 \pm 1^{\circ} \mathrm{C}$ with $50 \pm 5 \%$ humidity and illumination from 09:00 to 21:00. All animals were treated in accordance with national guidelines for the care and use of laboratory animals (Notification of the Prime Minister's Office of Japan). The Animal Experimental Committee of The University of Tokyo approved all experimental protocols.

During an acclimation period of approximately 1 week, the rats were allowed free access to water and a diet based on the AIN-93M formula. The rats were then separated into two groups matched for body weight and food efficiency. Each group was allowed free access to the experimental diet containing either LCTs or MCTs. Rats in the LCT group received the AIN-93M diet modified to contain 7\% corn oil $(\mathrm{w} / \mathrm{w})$; those in the MCT group received the same diet modified to contain $1 \%$ corn oil and $6 \%$ MCT oil $(\mathrm{w} /$ w). MCTs and LCTs (corn oil) were obtained from The Nisshin OilliO Group Ltd. (Tokyo, Japan). The compositions of the diet are presented in Table 1, while Table 2 shows the fatty acid compositions of the test lipids, as measured by gas chromatography ${ }^{15)}$. Each group of rats was allowed ad libitum access to the experimental diet, and their food intake and body weight were recorded every second day.
Table 1 Compositions of the experimental diets.

\begin{tabular}{lcc}
\hline \multirow{2}{*}{\multicolumn{1}{c}{ Ingredients }} & \multicolumn{2}{c}{ Dietary group $(\mathrm{g} / \mathrm{kg}$ diet $)$} \\
\cline { 2 - 3 } & LCT & MCT \\
\hline Cornstarch & 590.686 & 590.686 \\
Casein & 140 & 140 \\
Sucrose & 100 & 100 \\
LCTs & 70 & 10 \\
MCTs & - & 60 \\
Cellulose powder & 50 & 50 \\
Mineral mix (AIN-93M) & 35 & 35 \\
Vitamin mix (AIN-93) & 10 & 10 \\
L-Cystine & 1.8 & 1.8 \\
Choline bitartrate & 2.5 & 2.5 \\
tert-Butyl hydroquinone & 0.014 & 0.014 \\
\hline
\end{tabular}

LCTs, long-chain triacylglycerols (corn oil); MCTs, medium-chain triacylglycerols

Table 2 Fatty acid compositions of the test lipids.

\begin{tabular}{|c|c|c|}
\hline \multirow{2}{*}{ Fatty acid* } & LCTs & MCTs \\
\hline & \multicolumn{2}{|c|}{$\mathrm{g} / 100 \mathrm{~g}$ total fatty acids } \\
\hline $8: 0$ & ND & 59.8 \\
\hline 10:0 & ND & 22.2 \\
\hline 18:0 & 5.8 & 3.4 \\
\hline $18: 1$ & 37.6 & 9.3 \\
\hline $18: 2$ & 46.3 & 4.0 \\
\hline Others & 10.3 & 1.3 \\
\hline
\end{tabular}

LCTs, long-chain triacylglycerols (corn oil); MCTs, medium-chain triacylglycerols; ND, not detected; *, numbers are carbon atoms:double bonds

\subsection{Casting-induced hindlimb immobilization and tissue sampling}

After being fed the experimental diets for 3 days, unilateral hindlimb immobilization was performed on the left hindlimb as described previously ${ }^{10,11)}$. Briefly, the rats were anesthetized lightly with isoflurane for the attachment of the casting material. The left hindlimb was fixed in a shortened position with complete plantar flexion of the ankle joint, using casting tape (Scotchcast Plus-J; 3M Health Care, St. Paul, MN). The animals were checked daily for damage to the casting material, which was repaired as required. The rats were immobilized for 3, 7, and 14 days.

To determine total ketone body levels in the freely fed state in both groups, blood samples were collected into heparinized capillary tubes from the tail vein between 09:00 and 09:30 (immediately after the 12-h dark period) on day 3 of immobilization. The capillary tubes were then centrifuged and plasma samples were stored at $-80^{\circ} \mathrm{C}$ until 


\section{MCTs alleviate skeletal muscle atrophy}

analysis.

At the end of the immobilization period, the casts were removed under isoflurane anesthesia and the soleus muscle was dissected from both legs and weighed. During castinginduced hindlimb immobilization, anti-gravity muscles that cross a single joint, such as the soleus, are most vulnerable to atrophy ${ }^{16)}$. The muscle samples were frozen in liquid $\mathrm{N}_{2}$ and stored at $-80^{\circ} \mathrm{C}$ until analysis.

In a preliminary experiment, we confirmed that the contralateral soleus muscle in the nonimmobilized limb of the animals that underwent unilateral immobilization for 14 days did not undergo significant compensatory muscle hypertrophy (muscle mass and muscle protein content) in comparison with the left or right soleus muscle of animals that were not immobilized (data not shown).

\subsection{Muscle homogenization}

Frozen soleus muscle tissue was homogenized in icecold Radio-Immuno Precipitation Assay lysis buffer (EMD Millipore, Temecula, CA) containing $50 \mathrm{mM}$ Tris-HCl, $\mathrm{pH}$ 7.4, $150 \mathrm{mM} \mathrm{NaCl}, 0.25 \%$ deoxycholic acid, 1\% NP-40, 1 $\mathrm{mM}$ ethylenediaminetetraacetic acid, protease inhibitor cocktail (SIGMA-Aldrich, St. Louis, MO), and phosphatase inhibitors (PhosSTOP; Roche, Basel, Switzerland). The homogenates were frozen and thawed 3 times to disrupt intracellular organelles and rotated end-over-end at $4{ }^{\circ} \mathrm{C}$ for $60 \mathrm{~min}$ for protein solubilization. Total protein content per muscle was measured using a bicinchoninic acid (BCA) protein assay kit (Pierce, Rockford, IL). The homogenized samples were centrifuged at $700 \times g$ for $5 \mathrm{~min}$ at $4^{\circ} \mathrm{C}$ and the supernatants were harvested.

\subsection{Western blotting}

Protein concentrations of the supernatants were measured using a BCA protein assay kit. The samples were prepared in Laemmli sample buffer (Wako Pure Chemical, Osaka, Japan) and heated for $5 \mathrm{~min}$ in a heating block at $95^{\circ} \mathrm{C}$. Equal amounts of sample protein were subjected to sodium dodecyl sulfate-polyacrylamide gel electrophoresis $(7.5 \%, 10 \%$, or $15 \%$ resolving gels) and then transferred to polyvinylidene difluoride membranes at $220 \mathrm{~mA}$ for 90 min. After transfer, the membranes were blocked for $1 \mathrm{~h}$ at room temperature in Tris-buffered saline (TBS) with $0.1 \%$ Tween 20 (TBS-T; 20 mM Tris base, $137 \mathrm{mM} \mathrm{NaCl}, \mathrm{pH} 7.6$ ) supplemented with $5 \%(\mathrm{w} / \mathrm{v})$ nonfat powdered milk or $5 \%$ $(\mathrm{w} / \mathrm{v})$ bovine serum albumin (BSA). The membranes were incubated overnight at $4{ }^{\circ} \mathrm{C}$ with a primary antibody diluted 1:1000 in TBS-T containing 5\% BSA. The following primary antibodies were used: anti-microtubule-associated protein light chain 3 (LC3) (Medical \& Biological Laboratories, Nagoya, Japan), anti-muscle ring finger protein-1 (MuRF-1) (Abcam, Cambridge, MA), anti-Forkhead box class O (FoxO) 3a(Cell Signaling Technology, Danvers, MA), and anti-p50/p105 (Cell Signaling Technology). The membranes were then incubated for $1 \mathrm{~h}$ at room temperature with secondary antibodies (goat anti-rabbit IgG or goat anti-mouse IgG) diluted 1:5000 in TBS-T containing 1\% nonfat powdered milk. Bands were visualized using an enhanced chemiluminescence reagent (GE Healthcare Life Sciences, Piscataway, NJ) and quantified by Image Studio (LI-COR, Lincoln, NE). The membranes were stained with Ponceau (Sigma-Aldrich) to verify equal protein loading across lanes.

\subsection{Analysis of plasma total ketone body concentration}

Plasma total ketone body concentration was measured using a colorimetric assay (Autokit Total Ketone Bodies; Wako Pure Chemicals) as described previously ${ }^{15)}$.

\subsection{Statistical analysis}

Data for western blot assays in the immobilized soleus muscle were calculated and expressed as a percentage(\%) of the value of each contralateral non-casted muscle, while the values from the contralateral muscles were set at 100\% (Values of the contralateral muscle are indicated by dotted lines in each figure). Data are presented as the mean \pm standard error of the mean (SEM). Two-way analysis of variance (ANOVA; Social Survey Research Information Co., Ltd., Tokyo, Japan) was used to examine the effects of casting duration and diet conditions. When significant main effects and interactions were detected by ANOVA, posthoc comparisons were made using Fisher's LSD test. Statistical significance was defined as $p<0.05$.

\section{RESULTS}

\subsection{Body weight, food intake, and food efficiency}

As shown in Table 3, there were no significant differences in body weight, food intake, or food efficiency in the LCT- and MCT-fed rats.

\subsection{Plasma total ketone body concentration}

Plasma total ketone body concentration after 3 days of immobilization ( 6 days of dietary treatment) was significantly higher in the MCT group than in the LCT group (112 \pm 5 vs. $56 \pm 8 \mu \mathrm{mol} / \mathrm{L}$, respectively, $p<0.001)$.

\subsection{Muscle mass and total protein content}

Figure 1 shows the percentage change of muscle mass and total protein content of the immobilized soleus muscle compared with the contralateral non-casted muscle. Soleus muscle mass and total protein content in the immobilized muscle decreased significantly after immobilization for 3, 7, and 14 days.

Magnitude of muscle mass and total protein loss in the soleus muscle was significantly lower in the MCT group than in the LCT group (Fig. 1-A and -B). 


\section{S. Nishimura, M. Inai and T. Takagi et al.}

Table 3 Body weight, food intake, and food efficiency in rats.

\begin{tabular}{|c|c|c|c|c|c|c|}
\hline & \multicolumn{2}{|c|}{3 days } & \multicolumn{2}{|c|}{7 days } & \multicolumn{2}{|c|}{14 days } \\
\hline & LCT & MCT & LCT & MCT & LCT & $\mathrm{MCT}$ \\
\hline Body weight $(\mathrm{g})$ & $216.5 \pm 3.7$ & $219.1 \pm 2.0$ & $229.9 \pm 5.2$ & $226.7 \pm 4.1$ & $266.8 \pm 11.8$ & $263.0 \pm 4.9$ \\
\hline Food intake (g/day) & $16.4 \pm 0.2$ & $17.0 \pm 0.3$ & $15.6 \pm 0.2$ & $15.7 \pm 0.3$ & $17.0 \pm 0.5$ & $18.1 \pm 0.3$ \\
\hline Food efficiency $(\%) *$ & $21.0 \pm 0.9$ & $22.3 \pm 1.0$ & $10.1 \pm 1.4$ & $7.9 \pm 1.5$ & $12.2 \pm 2.5$ & $11.2 \pm 0.9$ \\
\hline
\end{tabular}

LCT, long-chain triacylglycerol diet group; MCT, medium-chain triacylglycerol diet group; Values are means $\pm \mathrm{SEM}$, $\mathrm{n}=6 .{ }^{*}$, equal to weight gain $(\mathrm{g}) /$ food intake $(\mathrm{g}) \times 100$.
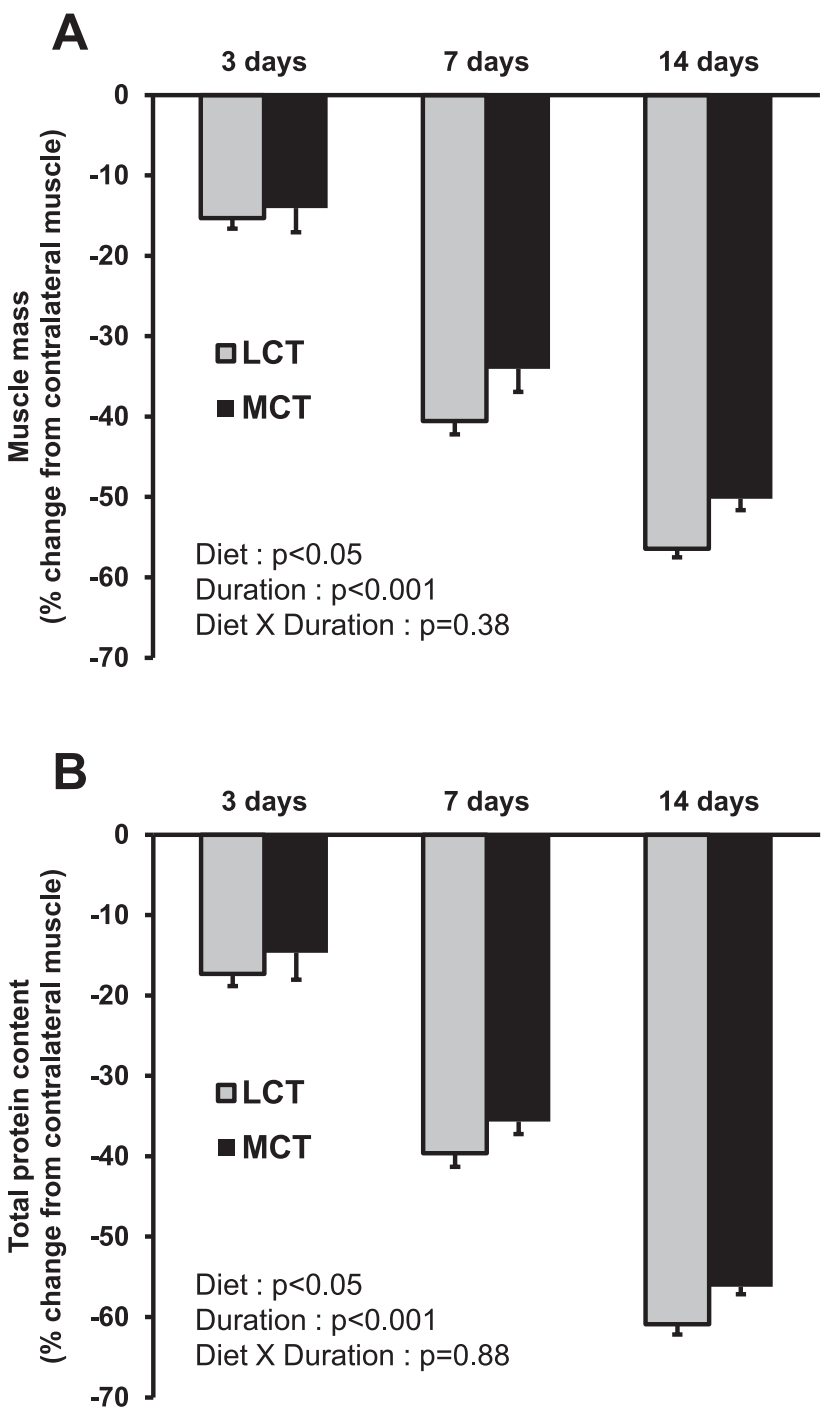

Fig. 1 Effects of the dietary intake of LCTs or MCTs on muscle mass (A) and total protein content (B) in the soleus muscle of rats subjected to unilateral immobilization. Data are expressed as the \% change from each contralateral non-casted muscle. Bars represent mean \pm SEM, $\mathrm{n}=6$. Differences were assessed by two-way ANOVA.

\subsection{LC3-I and -II content in soleus muscle}

The microtubule-associated protein LC3 is used widely to monitor the autophagy-lysosome system ${ }^{17)}$. The cytosolic form of LC3 (LC3-I) conjugates with phosphatidylethanolamine to form an LC3-phosphatidylethanolamine conjugate (LC3-II), which is recruited to autophagosomal membranes. As the amount of LC3-II is correlated with the extent of autophagosome formation and an increased LC3-II/LC3-I ratio is representative of accelerated autophagy-lysosome activity, we determined LC3-I and LC3-II expression levels in soleus muscle and used the LC3-II/LC3-1 ratio as a marker of autophagy-lysosome activity (Fig. 2 ${ }^{18)}$.

In both the LCT and MCT groups, unilateral immobilization increased LC3-II, but not LC3-I content, in the soleus muscle compared with the contralateral non-casted muscle, resulting in a higher LC3-II/LC3-I ratio in the immobilized muscle (Fig. 2-B and -C). The LC3-II content and LC3-II/LC3-I ratio in the immobilized soleus muscle relative to the contralateral muscle were not significantly different between the two groups (Fig. 2-C).

\subsection{MuRF-1 protein content in soleus muscle}

A chain of ubiquitin molecules is covalently attached to proteins to be degraded by the ubiquitin-proteasome pathway, targeting them for rapid degradation to short peptides by the $26 \mathrm{~S}$ proteasome ${ }^{19)}$. Ubiquitin-protein ligase (E3) is the key enzyme for attaching ubiquitin to protein substrates. The muscle-specific E3 ubiquitin ligase MuRF-1 is upregulated during muscle atrophy and is involved in the breakdown of myofibrillar proteins ${ }^{10,20)}$. In this study, we determined MuRF-1 protein levels in the soleus muscle as a marker of ubiquitin-proteasome activity.

Figure 3 shows MuRF-1 protein content in the immobilized soleus muscle relative to the contralateral non-casted muscle. In the LCT group, unilateral immobilization increased MuRF-1 protein levels in the soleus muscle relative to the contralateral muscle. This increase in MuRF-1 protein after immobilization was completely abolished in the MCT group and was significantly lower than that observed in the LCT group.

\subsection{FoxO and p50 protein content in soleus muscle}

Emerging evidence suggests that FoxOs and nuclear fac- 

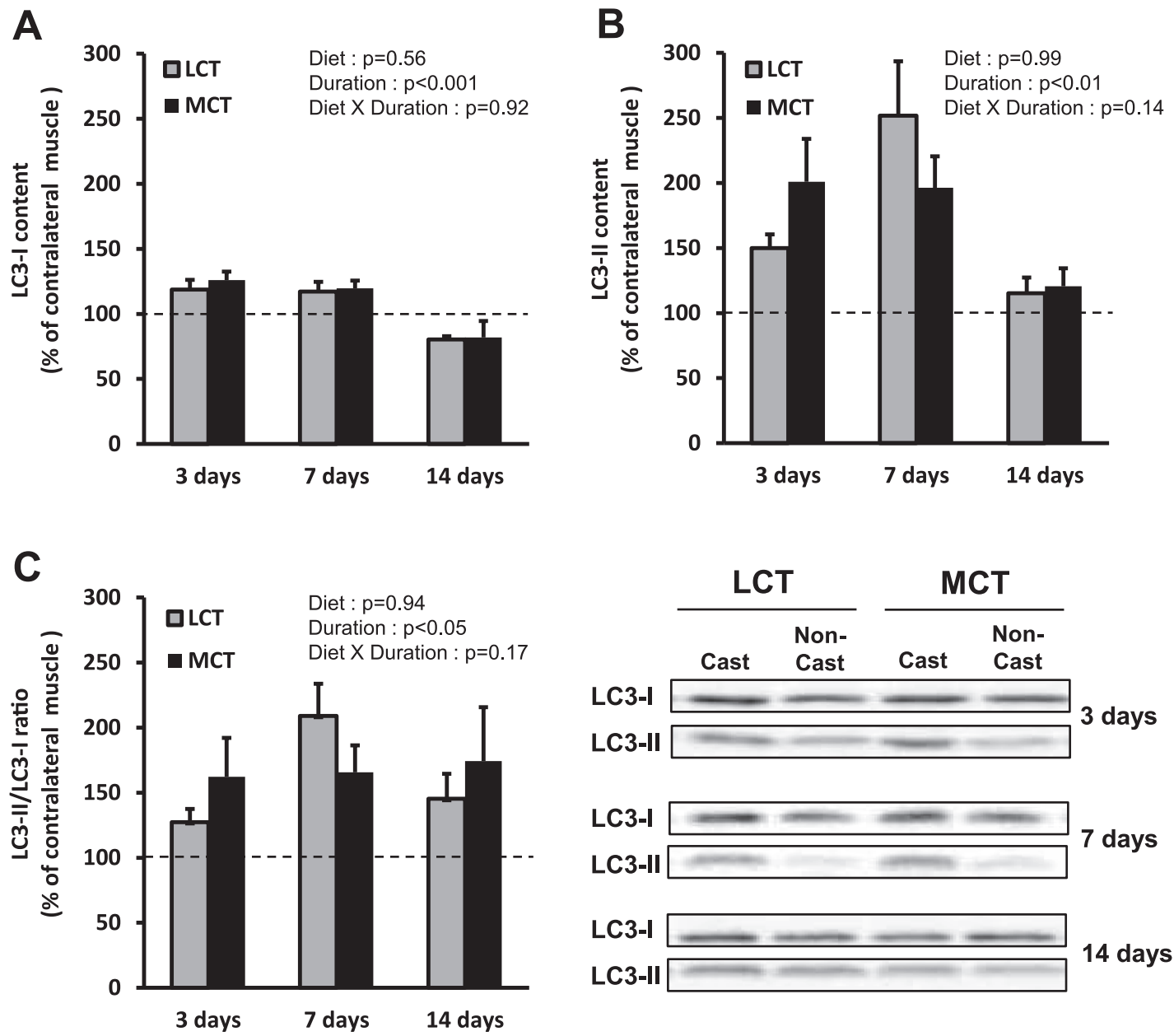

Fig. 2 Effects of the dietary intake of LCTs or MCTs on LC3-I(A) and LC3-II(B) levels and the LC3-II/LC3-I ratio(C)in the soleus muscle of rats subjected to unilateral immobilization. Data are shown as \% of each contralateral non-casted muscle. Bars represent mean $\pm \mathrm{SEM}, \mathrm{n}=6$. Dotted line indicates value of the contralateral muscle. Differences were assessed by two-way ANOVA. Cast, immobilized muscle with casting; Non-Cast, contralateral non-casted muscle.

tor-kappa $\mathrm{B}(\mathrm{NF}-\mathrm{\kappa} \mathrm{B})$ are important signaling pathways that are involved in the regulation of MuRF-1 expression and muscle atrophy ${ }^{21,22)}$. To elucidate the underlying mechanisms by which MCTs suppressed MuRF-1 content in immobilized soleus muscle, we determined the protein levels of the transcription factors FoxO3a and p50 (NF-кB1), which are upregulated during skeletal muscle atrophy ${ }^{10,23)}$. As shown in Fig. 4, no significant differences in FoxO3a and p50 protein levels in the immobilized soleus muscle relative to the contralateral non-casted muscle were observed between the LCT and MCT groups.

\section{DISCUSSION}

Previous studies have demonstrated that MCTs could ameliorate the malnutrition-induced reduction of serum albumin concentration $^{6-8)}$ and improve nitrogen balance after surgery ${ }^{5)}$, suggesting that MCTs exert favorable effects on protein metabolism. In this study, we examined the effects of the dietary intake of MCTs on muscle mass and protein content in rat soleus muscle during casting-induced immobilization, which causes substantial protein degradation. We found that MCT intake partially suppresses immobilization-induced atrophy in rat soleus muscle (Fig. 1). Our results may provide additional evidence that MCTs are a useful dietary treatment to rescue impaired protein metabolism.

While this paper was in preparation, we learned of a study by Abe et al. showing that the combined supplementation of MCTs, leucine, and vitamin D for 3 months increased muscle strength and function in frail elderly individuals $^{24)}$. They also demonstrated that these favorable effects were not observed in participants who received energy-matched LCTs with a leucine- and vitamin D-enriched supplement, suggesting that the improvements in muscle function and strength were due to the administration of MCTs. Our findings, together with those of Abe et al. ${ }^{24)}$, 

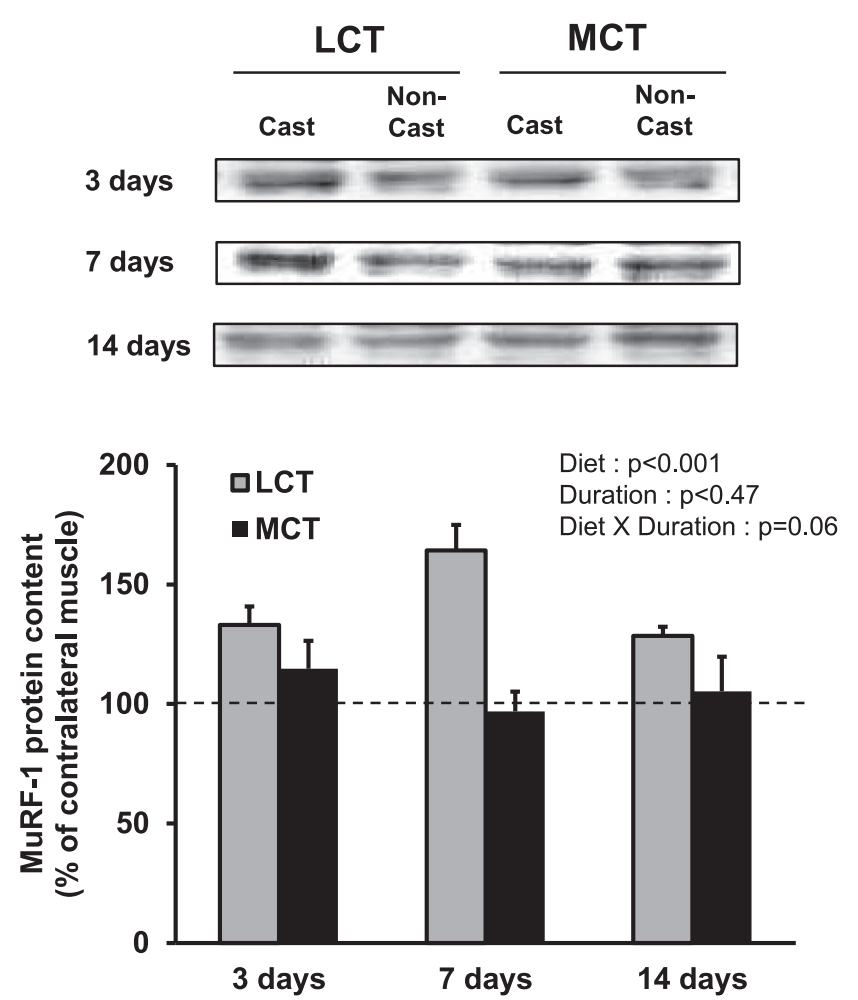

Fig. 3 Effects of the dietary intake of LCTs or MCTs on MuRF-1 protein levels in the soleus muscle of rats subjected to unilateral immobilization. Data are shown as \% of each contralateral non-casted muscle. Bars represent mean \pm SEM, $n=6$. Dotted line indicates value of the contralateral muscle. Differences were assessed by two-way ANOVA. Cast, immobilized muscle with casting; Non-Cast, contralateral non-casted muscle.

strongly support the possibility that the dietary intake of MCTs, rather than of LCTs, is an effective and feasible way to improve muscle mass as well as muscle strength and function under unhealthy conditions, although it remains to be determined whether MCTs are also effective in increasing muscle mass and strength in active and healthy individuals and athletes.

Faster protein degradation is an important factor in muscle atrophy induced by reduced mechanical loading ${ }^{11)}$. Under such conditions, the ubiquitin-proteasome pathway is thought to play a key role in degrading intracellular proteins, such as myofibrillar proteins. Two muscle-specific E3 ubiquitin ligases, atrophy gene-1 (Atrogin-1)/muscle atrophy F-box and MuRF-1, are upregulated in several muscle atrophy models such as casting, hindlimb suspension, and denervation, and are considered to be master regulators of muscle atrophy ${ }^{20)}$. However, accumulating evidence suggests that MuRF-1 is involved in the breakdown of myofibrillar proteins, whereas Atrogin-1 expression is a poor indicator of muscle protein degradation ${ }^{25)}$. In this context, we determined MuRF-1 protein content in the soleus muscle and found that the increase in MuRF-1 protein levels in the immobilized soleus muscle of the MCT group was completely abolished and was significantly lower than that observed in the LCT group (Fig. 3). These results suggest that the preventive effects of MCTs on muscle atrophy are mediated, at least in part, by inhibition of the ubiquitin-proteasome pathway.

The FoxO and NF-кB transcription factors are reported to be involved in the regulation of MuRF-1 gene expression in disuse-induced muscle atrophy ${ }^{21,22)}$. To further elucidate the mechanism underlying the suppression of MuRF-1 expression by MCTs, we determined FoxO3a and p50 $(\mathrm{NF}-\kappa \mathrm{B} 1)$ protein levels in the soleus muscle. However, contrary to our hypothesis, no significant difference in FoxO3a or p50 protein expression in the immobilized soleus muscle was observed between the LCT and MCT groups (Fig. 4). Therefore, it is likely that a mechanism other than the FoxOs or NF-кB pathway is responsible for the suppression of MuRF-1 expression by MCTs. Putative mechanisms for the MCT-induced suppression of MuRF-1 expression are ketone bodies and histone deacetylases (HDACs). MCT intake stimulates the production of hepatic ketone bodies, including $\beta$-hydroxybutyrate. Recent studies have shown that $\beta$-hydroxybutyrate has an inhibitory effect on HDAC activity ${ }^{26)}$, which is involved in the regulation of MuRF-1 expression ${ }^{27)}$. In the present study, we found that plasma total ketone body concentration was significantly higher in the MCT group than in the LCT group, suggesting that the dietary intake of MCTs may prevent the immobilized-induced increase in MuRF-1 content through the $\beta$-hydroxybutyrate-HDAC axis. Unfortunately, we could not determine HDAC activity in this study because the atrophied soleus muscles were very small and all of the tissue was used for western blot analyses, leaving no material for additional experiments. Future studies are needed to examine the effects of MCT intake on HDAC activity and its relationship with MuRF-1 expression in skeletal muscle.

In this study, the dietary intake of MCTs did not fully prevent immobilization-induced soleus muscle atrophy, despite the complete inhibition of MuRF-1 upregulation. The autophagy-lysosome pathway is another major protein degradation pathway in skeletal muscle. As shown in Fig. 2, the LC3-II/LC3-I ratio was increased in the immobilized soleus muscle relative to the contralateral non-casted muscle in both the MCT and LCT groups. Therefore, it appears that MCT intake has little effect on autophagic protein degradation in skeletal muscle during inactivity and failed to prevent muscle atrophy completely. 

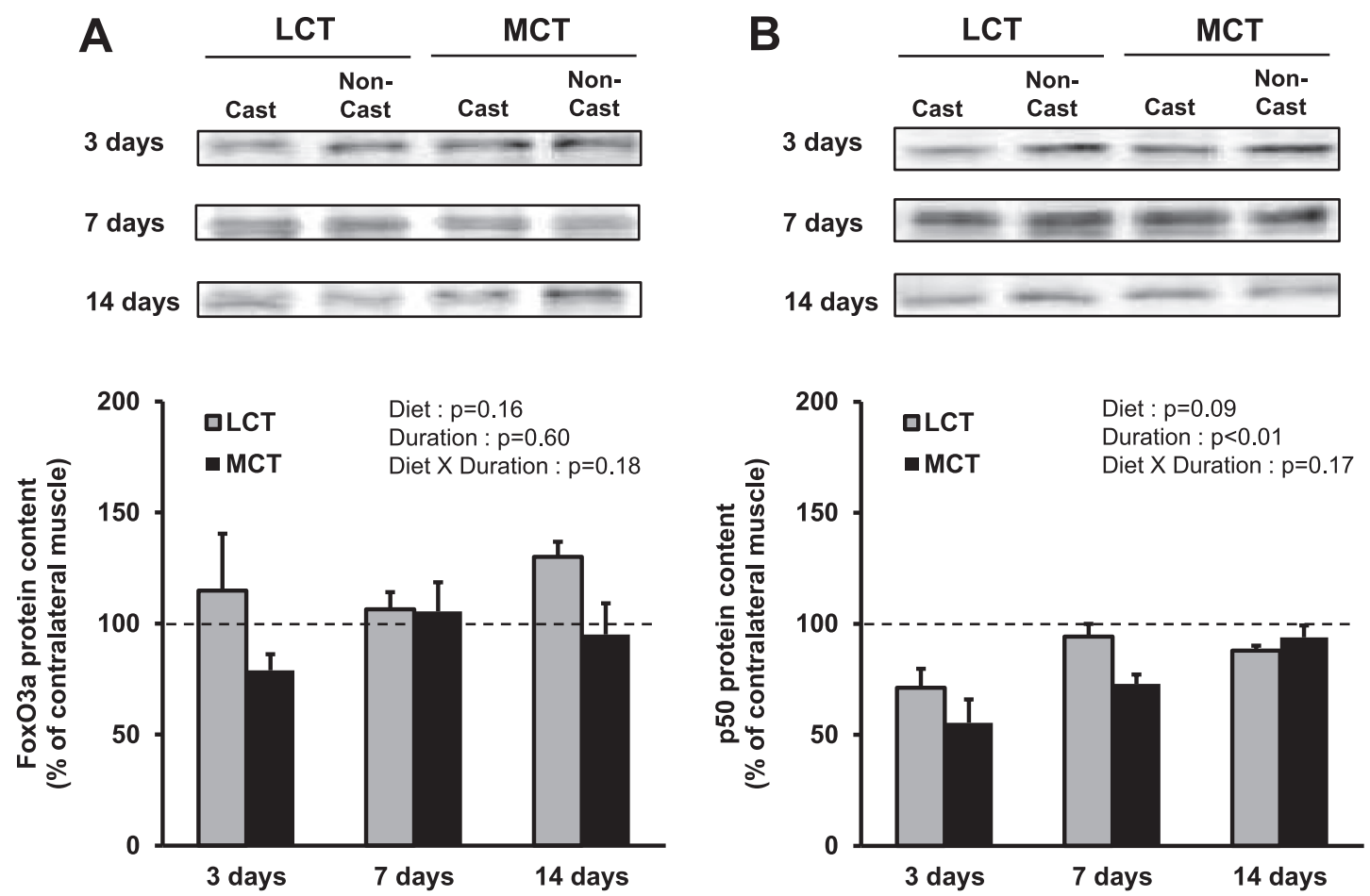

Fig. 4 Effects of the dietary intake of LCTs or MCTs on FoxO3a(A) and p50(B) protein levels in the soleus muscle of rats subjected to unilateral immobilization. Data are shown as \% of each contralateral non-casted muscle. Bars represent mean \pm SEM, $n=6$. Dotted line indicates value of the contralateral muscle. Differences were assessed by two-way ANOVA. Cast, immobilized muscle with casting; Non-Cast, contralateral non-casted muscle.

\section{CONCLUSION}

The dietary intake of MCTs was found to have preventive effects on muscle atrophy induced by immobilization. The mechanism responsible for the favorable effects of MCT appears to be, at least in part, suppression of the expression of the muscle-specific ubiquitin ligase MuRF-1.

\section{Acknowledgements}

This work was supported by JSPS KAKENHI Grant Number JP15K01615(to S.T.).

\section{References}

1) Aoyama, T.; Nosaka, N.; Kasai, M. Research on the nutritional characteristics of medium-chain fatty acids. $J$. Med. Invest. 54, 385-388(2007).

2) Bach, A.C.; Babayan, V.K. Medium-chain triglycerides: an update. Am. J. Clin. Nutr. 36, 950-962(1982).

3) Han, J.R.; Deng, B.; Sun, J.; Chen, C.G.; Corkey, B.E.; Kirkland, J.L.; Ma, J.; Guo, W. Effects of dietary medium-chain triglyceride on weight loss and insulin sensitivity in a group of moderately overweight free-living type 2 diabetic Chinese subjects. Metabolism 56, 985-
991 (2007)

4) Eckel, R.H.; Hanson, A.S.; Chen, A.Y.; Berman, J.N.; Yost, T.J.; Brass, E.P. Dietary substitution of mediumchain triglycerides improves insulin-mediated glucose metabolism in NIDDM subjects. Diabetes 41, 641-647 (1992).

5) Jiang, Z.M.; Zhang, S.Y.; Wang, X.R.; Yang, N.F.; Zhu, Y.; Wilmore, D. A comparison of medium-chain and longchain triglycerides in surgical patients. Ann. Surg. 217, 175-184 (1993).

6) Kojima, K.; Ogawa, A.; Nakamura, R.; Kasai, M. Effect of dietary medium-chain triacylglycerol on serum albumin and nitrogen balance in malnourished rats. $J$. Clin. Biochem. Nutr. 42, 45-49 (2008).

7) Nosaka, N.; Adachi, K.; Kawashima, Y.; Suzuki, H.; Hayashi, S.; Aoyama, T.; Nakamura, T. Effect of ingestion of medium-chain fatty acids on serum albumin in the elderly with protein-energy malnutrition (PEM). J. Jpn. Soc. Clin. Nutr. 32, 52-61 (2010).

8) Sekine, S.; Terada, S.; Aoyama, T. Medium-chain triacylglycerol suppresses the decrease of plasma albumin level through the insulin-Akt-mTOR pathway in the livers of malnourished rats. J. Nutr. Sci. Vitaminol. (Tokyo) 59, 123-128(2013).

9) Booth, F.W. Time course of muscular atrophy during immobilization of hindlimbs in rats. J. Appl. Physiol. 
Respir. Environ. Exerc. Physiol. 43, 656-661(1977).

10) Okamoto, T.; Torii, S.; Machida, S. Differential gene expression of muscle-specific ubiquitin ligase MAFbx/ Atrogin-1 and MuRF1 in response to immobilizationinduced atrophy of slow-twitch and fast-twitch muscles. J. Physiol. Sci. 61, 537-546(2011).

11) Krawiec, B.J.; Frost, R.A.; Vary, T.C.; Jefferson, L.S.; Lang, C.H. Hindlimb casting decreases muscle mass in part by proteasome-dependent proteolysis but independent of protein synthesis. Am. J. Physiol. Endocrinol. Metab. 289, E969-E980 (2005).

12) Rock, K.L.; Gramm, C.; Rothstein, L.; Clark, K.; Stein, R.; Dick, L.; Hwang, D.; Goldberg, A.L. Inhibitors of the proteasome block the degradation of most cell proteins and the generation of peptides presented on MHC class I molecules. Cell 78, 761-771 (1994).

13) Mortimore, G.E.; Pösö, A.R. Intracellular protein catabolism and its control during nutrient deprivation and supply. Annu. Rev. Nutr. 7, 539-564(1987).

14) Terada, S.; Sekine, S.; Aoyama, T. Dietary Intake of Medium- and Long-chain Triacylglycerols Prevents the Progression of Hyperglycemia in Diabetic ob/ob Mice. J. Oleo Sci. 64, 683-688(2015).

15) Nonaka, Y.; Takagi, T.; Inai, M.; Nishimura, S.; Urashima, S.; Honda, K.; Aoyama, T.; Terada, S. Lauric Acid Stimulates Ketone Body Production in the KT-5 Astrocyte Cell Line. J. Oleo Sci. 65, 693-699(2016).

16) You, J.S.; Park, M.N.; Song, W.; Lee, Y.S. Dietary fish oil alleviates soleus atrophy during immobilization in association with Akt signaling to p70s6k and E3 ubiquitin ligases in rats. Appl. Physiol. Nutr. Metab. 35, 310-318 (2010).

17) Mizushima, N.; Yoshimori, T. How to interpret LC3 immunoblotting. Autophagy 3, 542-545 (2007).

18) Lee, J.H.; Lee, J.H.; Jin, M.; Han, S.D.; Chon, G.R.; Kim, I.H.; Kim, S.; Kim, S.Y.; Choi, S.B.; Noh, Y.H. Diet control to achieve euglycemia induces significant loss of heart and liver weight via increased autophagy compared with ad libitum diet in diabetic rats. Exp. Mol. Med. 46, e111 (2014).

19) Tisdale, M.J. The ubiquitin-proteasome pathway as a therapeutic target for muscle wasting. J. Support. Oncol. 3, 209-217(2005).
20) Bodine, S.C.; Latres, E.; Baumhueter, S.; Lai, V.K.; Nunez, L.; Clarke, B.A.; Poueymirou, W.T.; Panaro, F.J.; Na, E.; Dharmarajan, K.; Pan, Z.Q.; Valenzuela, D.M.; DeChiara, T.M.; Stitt, T.N.; Yancopoulos, G.D.; Glass, D.J. Identification of ubiquitin ligases required for skeletal muscle atrophy. Science 294, 1704-1708 (2001).

21) Milan, G.; Romanello, V.; Pescatore, F.; Armani, A.; Paik, J.H.; Frasson, L.; Seydel, A; Zhao, J.; Abraham, R.; Goldberg, A.L.; Blaauw, B.; DePinho, R.A.; Sandri, M. Regulation of autophagy and the ubiquitin-proteasome system by the FoxO transcriptional network during muscle atrophy. Nat. Commun. 6, 6670-6683 (2015).

22) Li, H.; Malhotra, S.; Kumar, A. Nuclear factor-kappa B signaling in skeletal muscle atrophy. J. Mol. Med. (Berl). 1113-1126 (2008).

23) Hunter, R.B.; Stevenson, E.; Koncarevic, A.; MitchellFelton, H.; Essig, D.A.; Kandarian, S.C. Activation of an alternative NF-kappaB pathway in skeletal muscle during disuse atrophy. FASEB J. 16, 529-538(2002).

24) Abe, S.; Ezaki, O.; Suzuki, M. Medium-chain triglycerides in combination with leucine and vitamin D increase muscle strength and function in frail elderly adults in a randomized controlled trial. J. Nutr. 146, 1017-1026 (2016).

25) Attaix, D.; Baracos, V.E. MAFbx/Atrogin-1 expression is a poor index of muscle proteolysis. Curr. Opin. Clin. Nutr. Metab. Care 13, 223-224(2010).

26) Shimazu, T.; Hirschey, M.D.; Newman, J.; He, W.; Shirakawa, K.; Le Moan, N.; Grueter, C.A.; Lim, H.; Saunders, L.R.; Stevens, R.D.; Newgard, C.B.; Farese, R.V. Jr.; de Cabo, R.; Ulrich, S.; Akassoglou, K.; Verdin, E. Suppression of oxidative stress by $\beta$-hydroxybutyrate, an endogenous histone deacetylase inhibitor. Science 339, 211-214 (2013).

27) Moresi, V.; Williams, A.H.; Meadows, E.; Flynn, J.M.; Potthoff, M.J.; McAnally, J.; Shelton, J.M.; Backs, J.; Klein, W.H.; Richardson, J.A.; Bassel-Duby, R.; Olson, E.N. Myogenin and class II HDACs control neurogenic muscle atrophy by inducing E3 ubiquitin ligases. Cell 143, 35-45(2010). 